\title{
Calcium Homeostasis in Human Induced Pluripotent Stem Cell-Derived Cardiomyocytes
}

\author{
Yee-Ki Lee • Kwong-Man Ng • Wing-Hon Lai • \\ Yau-Chi Chan • Yee-Man Lau • Qizhou Lian • \\ Hung-Fat Tse $\cdot$ Chung-Wah Siu
}

Published online: 26 May 2011

(C) The Author(s) 2011. This article is published with open access at Springerlink.com

\begin{abstract}
Rationale Cardiomyocytes generated from human induced pluripotent stem cells (hiPSCs) are suggested as the most promising candidate to replenish cardiomyocyte loss in regenerative medicine. Little is known about their calcium homeostasis, the key process underlying excitation-contraction coupling. Objective We investigated the calcium handling properties of hiPSC-derived cardiomyocytes and compared with those from human embryonic stem cells (hESCs).

Methods and Results We differentiated cardiomyocytes from hiPSCs (IMR90 and KS1) and hESCs (H7 and HES3) with established protocols. Beating outgrowths from embryoid bodies were typically observed 2 weeks after induction. Cells in these outgrowths were stained positively for tropomyosin and sarco-
\end{abstract}

\section{Subject codes \\ [136] Calcium cycling/excitation-contraction coupling, and [137] Cell biology/structural biology}

Yee-Ki Lee and Kwong-Man Ng contribute equally to this work.

Electronic supplementary material The online version of this article (doi:10.1007/s12015-011-9273-3) contains supplementary material, which is available to authorized users.

Y.-K. Lee · K.-M. Ng • W.-H. Lai · Y.-C. Chan • Y.-M. Lau •

Q. Lian $\cdot$ H.-F. Tse $\cdot$ C.-W. Siu $(\bowtie)$

Cardiology Division, Department of Medicine,

Queen Mary Hospital, the University of Hong Kong,

Hong Kong, China

e-mail: cwdsiu@hkucc.hku.hk

Y.-K. Lee $\cdot$ K.-M. Ng • W.-H. Lai • Y.-C. Chan • Y.-M. Lau •

Q. Lian $\cdot$ H.-F. Tse $\cdot$ C.-W. Siu

Research Center of Heart, Brain, Hormone and Healthy Aging, Li

Ka Shing Faculty of Medicine, the University of Hong Kong,

Hong Kong, China

Q. Lian

Eye Institute, Li Ka Shing Faculty of Medicine,

the University of Hong Kong,

Hong Kong, China meric alpha-actinin. Reverse-transcription polymerase chain reaction studies demonstrated the expressions of cardiacspecific markers in both hiPSC- and hESC-derived cardiomyocytes. Calcium handling properties of 20-day-old hiPSC- and hESC-derived cardiomyocytes were investigated using fluorescence confocal microscopy. Compared with hESC-derived cardiomyocytes, spontaneous calcium transients from both lines of hiPSC-derived cardiomyocytes were of significantly smaller amplitude and with slower maximal upstroke velocity. Better caffeine-induced calcium handling kinetics in hESC-CMs indicates a higher sacroplasmic recticulum calcium store. Furthermore, in contrast with hESC-derived cardiomyocytes, ryanodine did not reduce the amplitudes, maximal upstroke and decay velocity of calcium transients of hiPSC-derived cardiomyocytes. In addition, spatial inhomogeneity in temporal properties of calcium transients across the width of cardiomyocytes was more pronounced in hiPSC-derived cardiomyocytes than their hESC counterpart as revealed line-scan calcium imaging. Expressions of the key calcium-handling proteins including ryanodine recptor-2 (RyR2), sacroplasmic recticulum calcium-ATPase (SERCA), junction (Jun) and triadin (TRDN), were significantly lower in hiPSC than in hESCs.

Conclusions The results indicate the calcium handling properties of hiPSC-derived cardiomyocytes are relatively immature to hESC counterparts.

Keywords Human induced pluripotent stem cells . Cardiomyocytes $\cdot$ Calcium handling

\section{Introduction}

Human induced pluripotent stem cells (hiPSCs) can be generated from adult human dermal fibroblasts by transduction of a defined set of transcription factors to reprogram them back to an equivalent of the early embryonic state [1-4]. hiPSCs, 
resembling human embryonic stem cells (hESCs), possess remarkable self-renewal capacity and unquestioned potential to differentiate into genuine cardiomyocytes $[5,6]$. Being genetically identical to donor patients, patient-specific iPSCs avoid potential immune rejection and do not have the ethical issues specific to hESCs [3], thereby representing an attractive cell source for future cardiac regenerative therapy [7]. However, the prerequisite to the ultimate clinical application is that hiPSC-derived cardiac derivatives display normal physiological characteristics. Although the electrophysiological properties of hiPSC-derived cardiomyocytes have been reported $[5,6,8,9]$, which are similar to those of hESCderived cardiomyocytes and early fetal cardiomycoytes [7, 10-12], there is paucity of data concerning the calcium handling properties of hiPSC-derived cardiomyocytes, the key process underlying the excitation-contraction coupling.

In adult cardiomyocytes, a small calcium influx through Ltype calcium channels during systole triggers a large calcium release from the internal calcium store, sarcoplasmic reticulum (SR) through ryanodine receptors (RyR) [13]. Calciuminduced calcium release (CICR) is the primary mechanism linking electrical excitation and mechanical contraction in cardiomyocytes. During diastole, calcium is actively removed out of cytosol, mainly through SR calcium-ATPase (SERCA) back into SR and sodium/calcium exchanger out of cells [13]. It has been reported that ESC-derived cardiomyocytes exhibit immature calcium dynamics due to the underdeveloped SR and the developmental expression profiles of calcium handling proteins $[10,11,14-18]$. However, it remains unclear whether hiPSC-derived cardiomyocytes exhibit similar immaturity in calcium handling as hESC-derived cardiomyocytes. In fact, such immaturity may not only result in poor host-graft electromechanical integration when transplanted into recipient heart, but also lead to lethal arrhythmias. In this study, we examined the calcium handling properties of two hiPSC(IMR90- and KEVIN-SIU (KS)1-hiPSCs) derived cardiomyocytes with confocal microscopy in comparison with two hESC lines (H7- and HES3-hESCs).

\section{Methods}

hiPSC and hESC Culture and Cardiac Differentiation

We used hiPSC line, IMR90-iPSCs (iPS (IMR90)-1, clone 1, WiCell) (WiCell Research Institute, Madison, Wisconsin), KS1-iPSCs as generated by our group in feeder-free condition [19], and human embryonic stem cell (hESC) line H7- and HES3-hESCs (National Institutes of Health, NIH code WA07 \& ES03 respectively) (WiCell Research Institute, Madison, Wisconsin) for these following experiments. Undifferentiated cells were cultured on Matrigel ${ }^{\mathrm{TM}}$ (BD Biosciences, MA)-coated dishes with $\mathrm{mTeSR}^{\mathrm{TM}}$ medium
(Stem Cell Technologies, BC, Canada). Cardiac differentiation was performed according to a published protocol [20]. Briefly, cells were dissociated into clumps using $1 \mathrm{mg} / \mathrm{ml}$ Dispase (Invitrogen, CA, USA) and cultured in suspension using low attachment plates to form embryoid bodies. The embryoid bodies were transferred onto gelatin-coated plates in StemPro ${ }^{\circledR}$-34 medium (Invitrogen, CA, USA) supplemented with $2 \mathrm{mM}$ glutamine (Gibco, CA, USA), $4 \mathrm{mM}$ monothioglycerol (Sigma-Aldrich, St. Louis, MO), $50 \mu \mathrm{g} / \mathrm{ml}$ ascorbic acid (Sigma-Aldrich, St. Louis, MO) and $0.5 \mathrm{ng} / \mathrm{ml}$ BMP4 (R\&D). In addition, the medium was further supplemented with various cytokines according to the following sequence: post-plating day 1 to $4,10 \mathrm{ng} / \mathrm{ml}$

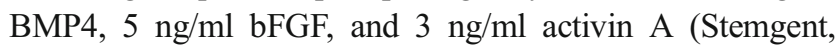
CA, USA); post-plating day 4 to $8,150 \mathrm{ng} / \mathrm{ml}$ DKK1 (Gibco BRL, Karlsruhe, Germany) and $10 \mathrm{ng} / \mathrm{ml}$ VEGF; and after day $8,150 \mathrm{ng} / \mathrm{ml}$ DKK1, $10 \mathrm{ng} / \mathrm{ml}$ VEGF (Peprotech, NJ, USA), and $5 \mathrm{ng} / \mathrm{ml} \mathrm{bFGF} \mathrm{(Peprotech,} \mathrm{NJ,} \mathrm{USA).}$

Isolation of hiPSC- and hESC-derived Cardiomyocytes

Differentiated hiPSC- and hESC-embryoid bodies containing beating outgrowths were micro-surgically dissected using a glass knife on post-plating day 21 as previously described [14, 21-23]. Beating cells were dissociated in medium containing $1 \mathrm{mg} / \mathrm{ml}$ collagenase B and $60 \mathrm{U} / \mathrm{ml}$ DNase I (Roche Applied Sciences Penzberg, Germany) at $37^{\circ} \mathrm{C}$ for $30 \mathrm{~min}$ and were re-suspended in Kraftbrühe (KB) solution containing $85 \mathrm{mM}$ $\mathrm{KCl}, 30 \mathrm{mM} \mathrm{K}_{2} \mathrm{HPO}_{4}, 5 \mathrm{mM} \mathrm{MgSO}$, 1 mM EGTA, $2 \mathrm{mM}$ $\mathrm{Na}_{2}$-ATP, $5 \mathrm{mM}$ pyruvic acid, $5 \mathrm{mM}$ creatine, $20 \mathrm{mM}$ taurine, and $20 \mathrm{mM}$ D-glucose at room temperature for $1 \mathrm{~h}$. Cells were then plated onto $0.1 \%$ gelatin-coated glass coverslips and maintained with culture medium.

Measurement of Cytosolic $\left[\mathrm{Ca}^{2+}\right]$

Cytosolic calcium transients were estimated in isolated cells using a confocal imaging system (Olympus Fluoview System version 4.2 FV300 TIEMPO) mounted on an upright Olympus microscope (IX71) as previously described [14, $16,17,24,25]$. Briefly, cells were loaded with 1:1 $(v / v)$ amount of 20\% Pluronic ${ }^{\circledR}$-F127 (Invitrogen, life technologies) and $5 \mu \mathrm{M}$ Fluo-3 AM (Sigma-Aldrich, St. Louis, MO) dissolved in DMSO with stock concentration of $5 \mathrm{mM}$ for $45 \mathrm{~min}$ at $37^{\circ} \mathrm{C}$ in Tyrode solution containing $140 \mathrm{mM} \mathrm{NaCl}$, $5 \mathrm{mM} \mathrm{KCl}_{1} 1 \mathrm{mM} \mathrm{MgCl}_{2}, 1.8 \mathrm{mM} \mathrm{CaCl}_{2}, 10 \mathrm{mM}$ glucose and $10 \mathrm{mM}$ HEPES at pH 7.4 [14]. Calcium transients of single cardiomyocytes were recorded with a temporal resolution of the line scan at 274 frames per second. Sacroplasmic reticular (SR) calcium load was estimated from peak calcium release after the addition of caffeine $(10 \mathrm{mM})$. All confocal calcium imaging experiments were performed within $48 \mathrm{~h}$ after isolation in order to minimize contamination of time- 
dependent changes in calcium handling property in culture. Raw data of fluorescence intensity was recorded by area vs. time mode (XYT) as a line plot, the calibration curve showed that there is linear relationship between fluo-3 intensity recorded with calcium concentration up to $630 \mathrm{nM}\left[\mathrm{Ca}^{2+}\right]$. Regarding to line scan images, they are recorded in line vs. time mode (XT). The data were then quantified as the background subtracted fluorescence intensity changes normalized to the background subtracted baseline fluorescence using Image J. Amplitudes, maximal upstroke and decay velocity of calcium transient were analyzed by Clampfit version 9.2.0.09. (Axon Instruments, Inc, Foster City, CA).

Reverse Transcription Polymerase Chain

Reaction (RT-PCR)

Total RNA from 2-week old hiPSC- and hESC-embryoid bodies was extracted with illustra RNAspin Mini kit (GE Healthcare, Buckinghamshire, UK). Reverse transcription was then performed using $0.5 \mu \mathrm{g}$ RNA in a final volume of $20 \mu \mathrm{l}$, using QuantiTect ${ }^{\mathbb{B}}$ reverse transcription kit (Qiagen, Hilden, Germany, http://www1.qiagen.com) according to the manufacturer's instructions. Details of a panel of cardiacspecific genes and calcium handling protein genes are summarized in Table 1. Quantitative PCR analysis was performed with real-time PCR Detector (Opticon 2 DNA Engine, MJ Research, Minnesota, USA) using the iQ SYBR Green Supermix (Bio-Rad Laboratories, Hercules, CA). For amplification, after initial holds for $5 \mathrm{~min}$ at $95^{\circ} \mathrm{C}, 50$ cycles of $95^{\circ} \mathrm{C}$ for $15 \mathrm{~s}$ followed by corresponding annealing temperature for $30 \mathrm{~s}$ and $72^{\circ} \mathrm{C}$ for $30 \mathrm{~s}$, melt curve analysis was performed. The relative quantification of PCR products was performed according to the $2^{-\Delta \Delta \mathrm{Ct}}$ method, using mouse GAPDH as internal control. Where $\Delta \Delta \mathrm{Ct}=\left[\left(\mathrm{Ct}_{\text {target gene }}-\mathrm{Ct}_{\mathrm{GAPDH}}\right)_{\mathrm{IMR} 90-\mathrm{iPS} \text { group }}-\left(\mathrm{Ct}_{\text {target gene }}-\right.\right.$ $\left.\left.\mathrm{Ct}_{\mathrm{GAPDH}}\right)_{\mathrm{H} 7 \mathrm{hES} \text { group }]}\right]$.

Statistical Analysis

Continuous variables are expressed as mean \pm standard deviation. Statistical comparisons were performed using Student's $t$ test. Calculations were performed with SPSS (version 14.0). A $p$-value $<0.05$ was considered statistically significant.

For additional methodological details, see the expanded methods section in the data supplement.

\section{Results}

Culture and Cardiac Differentiation of hiPSCs

Undifferentiated IMR-90 and KS1-hiPSCs were maintained in a feeder-free culture condition as previously
Table 1 Primers used for Real-time quantitative RT-PCR

\begin{tabular}{lll}
\hline Gene & Direction & Sequence $\left(5^{\prime}\right.$ to $\left.3^{\prime}\right)$ \\
\hline Nkx2.5 & Forward & TTCCCGCCGCCCCCGCCTTCTAT \\
& Reverse & CGCTCCGCGTTGTCCGCCTCTGT \\
$\alpha$-MHC & Forward & GTTGGTGTTGGCTTGCTCCTC \\
& Reverse & ATCAAGGAGCTCACCTACCAG \\
$\beta$-MHC & Forward & TGGGGCTTTGCTGGCACCTCC \\
& Reverse & GCGGAGGAGCAAGCCAACACC \\
NCX1 & Forward & TGTGCATCTCAGCAATGTCA \\
& Reverse & TGATGCCAATGCTCTCACTC \\
RYR2 & Forward & CGTTCTAACCAGCATCTCATC \\
& Reverse & CGAGCAATACAACCTGACC \\
SERCA2a & Forward & ACCCACATTCGAGTTGGAAG \\
& Reverse & CAGTGGGTTGTCATGAGTGG \\
CASQ2 & Forward & GAGCTTGTGGCCCAGGTCCT \\
& Reverse & GATCTCCACTGGGTCTTCAA \\
Junctin & Forward & GTAAAATGGCATCCCGAGAC \\
& Reverse & GGATGATGATGCCAGAGC \\
Triadin & Forward & TCAGTTGCTCCACACTGAGC \\
& Reverse & CCCATTTACAGACGGGAAAC \\
GAPDH & Forward & AGCCACATCGCTCAGACACC \\
& Reverse & GTACTCAGCGCCAGCATCG \\
\hline \multirow{2}{*}{ F } & &
\end{tabular}

$N k x 2.5$ NK2 transcription factor related, locus 5; $\alpha-M H C$ alphamyosin heavy chain; $\beta-M H C$ beta-myosin heavy chain; $N C X 1$ sodium calcium exchanger-1; RyR2 ryanodine receptor 2; SERCA-2a sarcoplasmic reticulum calcium ATPase 2a; CASQ2 calsequestrin-2; GAPDH Glyceraldehyde 3-phosphate dehydrogenase

described (Fig. 1) [19, 26]. Resembling to undifferentiated H7- and HES3-hESCs, hiPSC colonies expressed a panel of pluripotency markers including Oct-4, Nanog, stage-specific embryonic antigen (SSEA)-4, and tumorrejection antigen (TRA)-1-60 (Fig. 1a to p). Spontaneous beating outgrowths were typically observed 14 days after cardiac differentiation induction in both hiPSC- and hESC-derived embryoid bodies (Supplemental video 1 and 2). In immunocytochemical analysis of the cardiacspecific proteins: $\alpha$-actinin and tropomyosin were abundantly expressed in IMR90- and KS1-hiPSC- and hESCderived cardiomyocytes (Fig. 2a-h). The percentages of nodal-like, atrial-like and ventricular-like cardiomyocytes were not different significantly between hiPSC- and hESC-derived cardiomyocytes (Fig. 2i \& j). Quantitative RT-PCR revealed that although the expressions of $\alpha$ MHC and Nkx2.5 in two different lines of hiPSC-derived cardiomyocytes were comparable to those of hESCdervied cardiomyocytes, $\beta$-MHC, the adult isoform, was expressed in much lower level in hiPSC-derived cardiomyocytes (Fig. 2k \&l). 


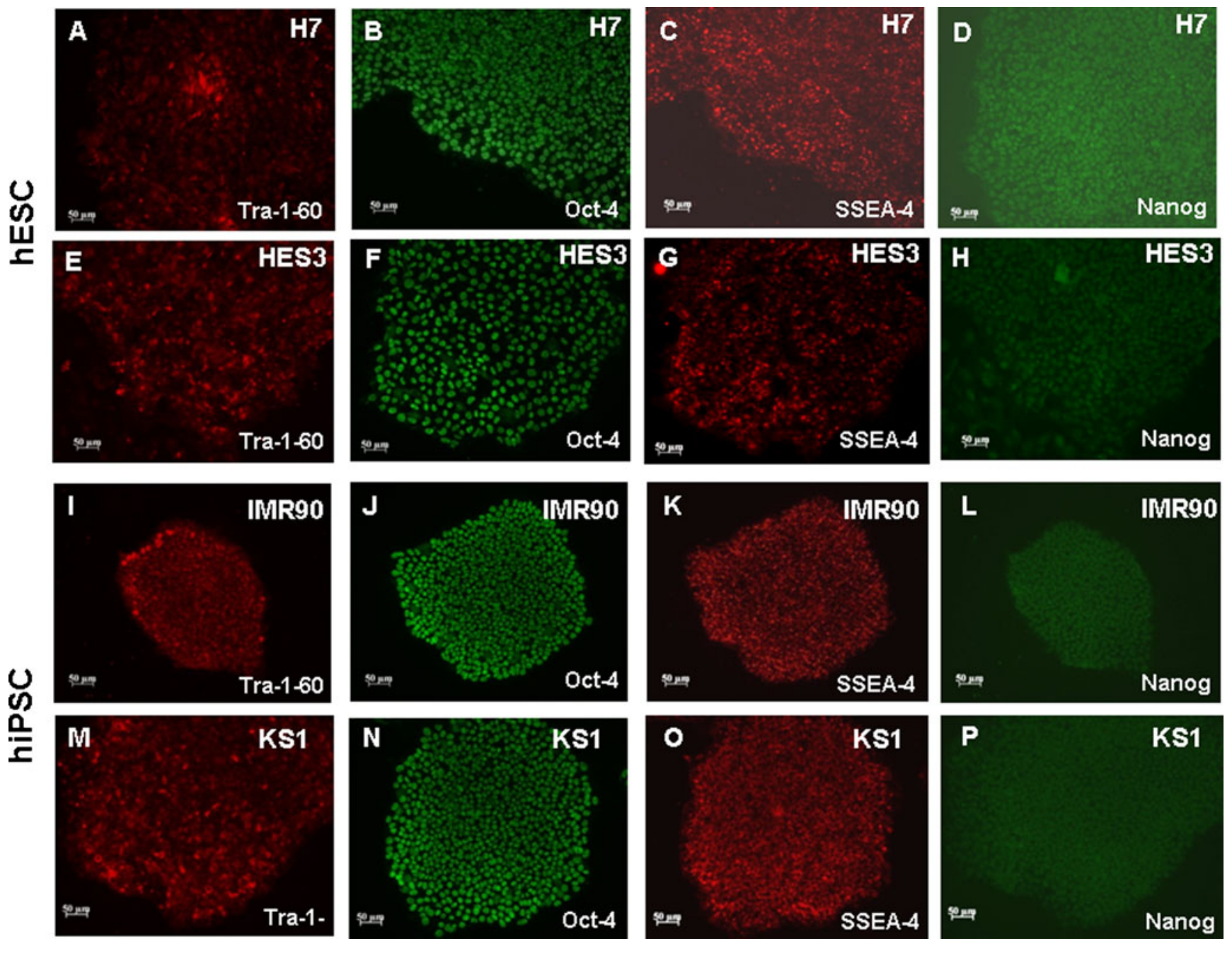

Fig. 1 Stem cell pluripotency markers expression in H7-, HES3-hESCs and IMR90-, KS1-hiPSCs. Tra-1-60 (a, e, i \& m), Oct4 (b, f, j \& n), SSEA-4 (c, g, k \& o) and Nanog $(\mathbf{d}, \mathbf{h}, \mathbf{l} \& \mathbf{p})$ and were expressed in both groups of cell lines

hiPSC-derived Cardiomyocytes Exhibit Immature Spontaneous Calcium Transient

We then evaluated the calcium handling properties of hiPSC-derived cardiomyocytes compared with those of human ESC-derived cardiomyocytes. Like hESC-derived cardiomyocytes, spontaneous rhythmic calcium transients were detected in hiPSC-derived cardiomycytes (Fig. 3a). However, hiPSC-derived cardiomyocytes exhibits more immature calcium handling properties than hESC counterparts (Fig. 3). Specifically, hiPSC-derived cardiomyocytes generated smaller amplitudes (IMR-90 iPSC-derived cardiomyocytes: $12.93 \pm 0.79 \mathrm{~F} / \mathrm{F}_{\mathrm{o}}$, and $\mathrm{KS} 1$-iPSC-derived cardiomyocytes: $9.10 \pm 0.78 \mathrm{~F} / \mathrm{F}_{\mathrm{o}}$, vs. H7-ESC-derived cardiomyocytes: $20.28 \pm 1.05 \mathrm{~F} / \mathrm{F}_{\mathrm{o}}$, and HES3-hESCderived cardiomyocytes: $15.79 \pm 1.25 \mathrm{~F} / \mathrm{F}_{\mathrm{o}} ; p<0.05$; Fig. 3b), slower maximal upstroke (IMR-90 iPSC-derived cardiomyocytes: $0.02 \pm 0.002 \mathrm{~F} / \mathrm{F}_{\mathrm{o}} / \mathrm{s}$, and KS1-iPSC-derived cardiomyocytes: $0.013 \pm 0.001 \mathrm{~F} / \mathrm{F}_{\mathrm{o}} / \mathrm{s}$, vs. H7-ESC-derived cardiomyocytes: $0.03 \pm 0.003 \mathrm{~F} / \mathrm{F}_{\mathrm{o}} / \mathrm{s}, \mathrm{HES} 3$-hESC-derived cardiomyocytes: $0.03 \pm 0.002 \mathrm{~F} / \mathrm{F}_{\mathrm{o}} / \mathrm{s} ; p<0.05$; Fig. $3 \mathrm{c}$ ), and significant slower maximal decay velocity (IMR-90 iPSCderived cardiomyocytes: $-0.018 \pm 0.001$, and KS1-iPSCderived cardiomyocytes: $-0.009 \pm 0.001 \mathrm{~F} / \mathrm{F}_{\mathrm{o}} / \mathrm{s}$, vs. H7ESC-derived cardiomyocytes: $-0.014 \pm 0.001$, and HES3hESC-derived cardiomyocytes: $0.019 \pm 0.002 \mathrm{~F} / \mathrm{F}_{\mathrm{o}} / \mathrm{s} ; p<0.05$; Fig. 3d) in comparison with hESC-derived cardiomyocytes.

Effects of Caffeine and Ryanodine on Calcium Transients of hiPSC-derived Cardiomyocytes

To determine whether SR in hiPSC-derived cardiomyocytes is in fact functional or not, we investigated the effects of caffeine, a RyR2 opener, and ryanodine, a RyR2 blocker, on the calcium transients of hiPSC-derived cardiomyocytes. Figure 4 shows that the application of caffeine $(10 \mathrm{mM})$ via a perfusion system induced a single large calcium transient that decayed slowly in both hiPSC-derived cardiomyocytes 


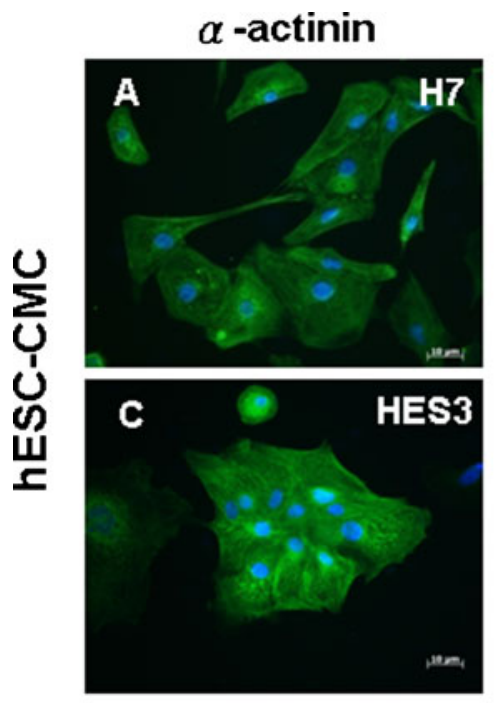

Tropomyosin
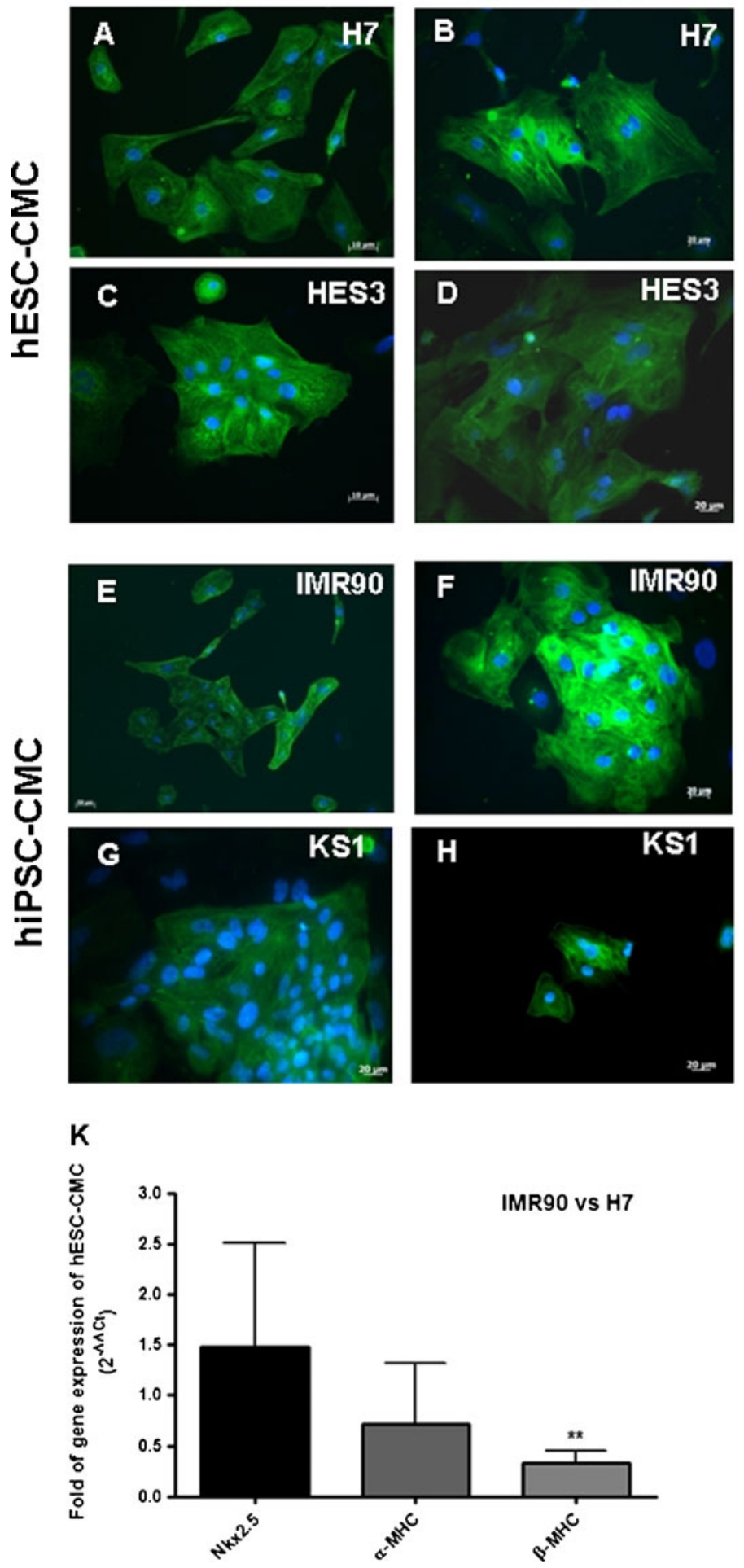

Fig. 2 Cardiomyocytes differentiated from H7-, HES3-hESCs and IMR90-, KS1-hiPSCs were stained with two typical cardiac markers, alpha-actinin (a, c, e \& g) and tropomyosin (b, d, f \& h). The proportion of nodal-like, atrial-like, and ventricular-like cells in hESCand hiPSC-derived cardiomyocytes as determined with action potential recordings with patch clamp (i \& j). Quantitation of gene

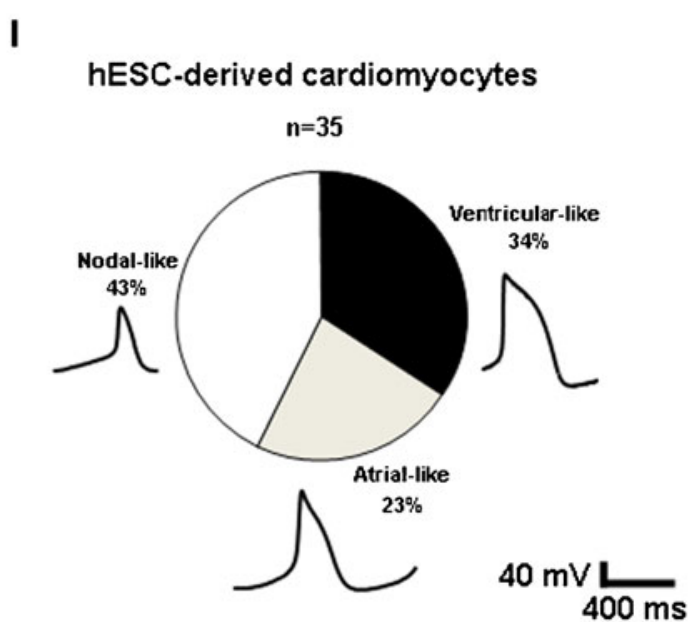

J hiPSC-derived cardiomyocytes
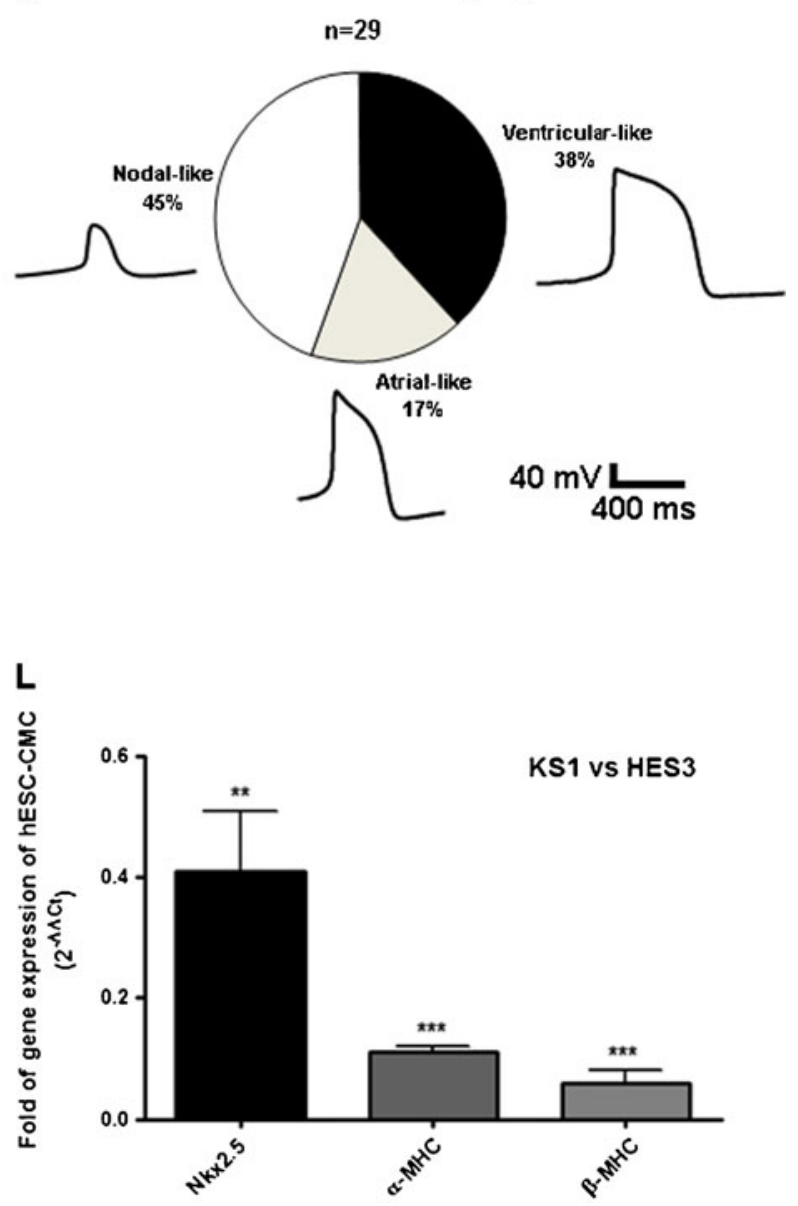

expression of cardiac proteins $(\mathbf{k} \& \mathbf{l})$ in differentiated $\mathrm{H} 7 \mathrm{hESCs}$ vs. IMR90-iPSCs and HES3 hESCs vs. KS1-iPSCs. Homeobox protein $\mathrm{Nkx}-2.5(\mathrm{Nkx} 2.5)$ and $\alpha / \beta$-myosin heavy chain $(\alpha / \beta$-MHC); Unpaired $t$-test was performed between $\mathrm{H} 7 \mathrm{hESC}$-CMCs and IMR90 iPSC-CMCs as well as HES3 hESC-CMCs and KS1 iPSC-CMCs $(n=3 ; * * p<0.005$ and $* * * p<0.0005)$ 
A

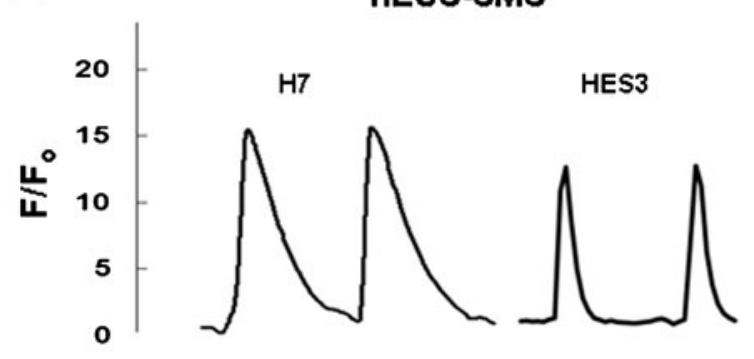

hiPSC-CMC

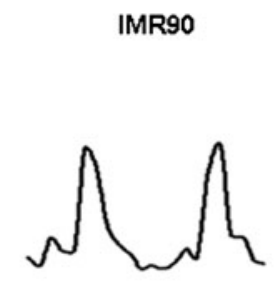

KS1
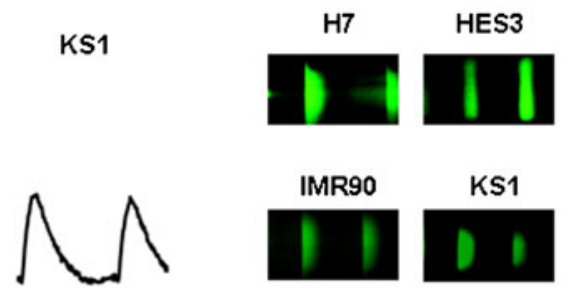

B

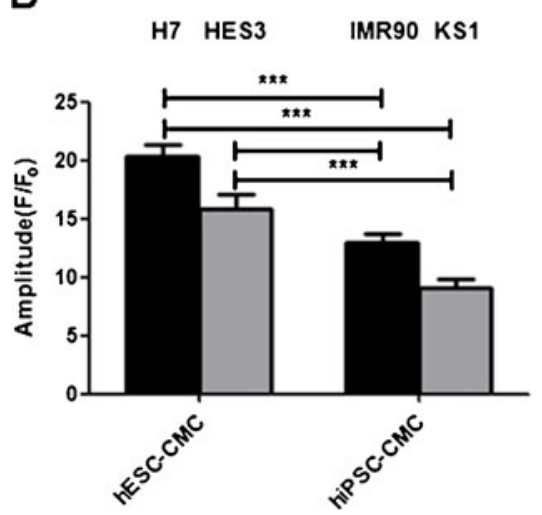

Fig. 3 Representative tracing of calcium transients and 2-D calcium transients in a $\mathrm{H} 7$ \& HES3 hESC derived cardiomyocytes (H7 \& HES3 hESC-CMCs), IMR90 \& KS1 hiPSC derived cardiomyocytes (IMR90 \& KS1 hiPSC-CMCs). b Amplitude, $\mathbf{c}$ maximal upstroke velocity ( $V_{\max }$ upstroke), and $\mathbf{d}$ maximal decay velocity ( $V_{\max }$ decay)

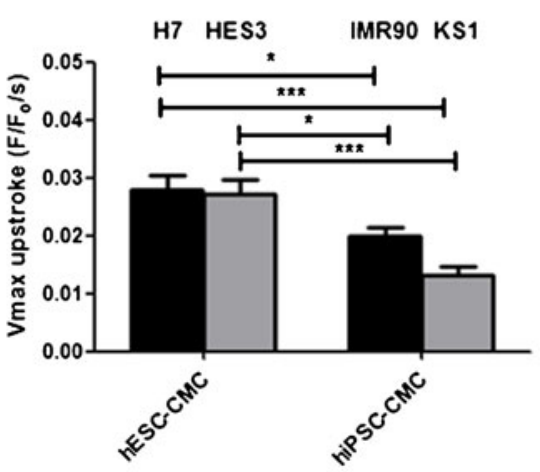

D

$10 \mathrm{~s}$

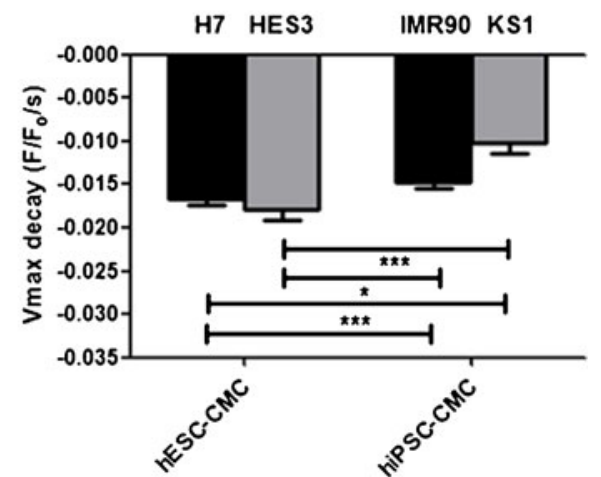

of calcium transients in the cardiomyocytes. Unpaired $t$-test was performed between $\mathrm{H} 7$ or HES3 hES-CMCs and the other two lines of iPS-CMCs $(n=10 ; n$ is corresponds to different individual cells derived from three different experiments) $\left({ }^{*} p<0.05, * * p<0.005\right.$ and $* * * p<0.0005)$

and hESC-derived cardiomyocytes (Fig. 4a). In comparison with hESC-derived cardiomyocytes, the caffeine-induced calcium transients in hiPSC-derived cardiomyocytes exhibited significantly smaller peak amplitudes (IMR-90 iPSC-derived cardiomyocytes: $9.36 \pm 1.14 \mathrm{~F} / \mathrm{F}_{\mathrm{o}}$, and KS1iPSC-derived cardiomyocytes: $3.39 \pm 0.57 \mathrm{~F} / \mathrm{F}_{\mathrm{o}}$, vs. H7ESC-derived cardiomyocytes: $21.23 \pm 0.65 \mathrm{~F} / \mathrm{F}_{\mathrm{o}}$, and HES3hESC-derived cardiomyocytes: $18.31 \pm 0.94 \mathrm{~F} / \mathrm{F}_{\mathrm{o}} ; p<0.05$, $n=10$; Fig. 4b) and a slower maximal delay velocity (IMR90 iPSC-derived cardiomyocytes: $0.01 \pm 0.002 \mathrm{~F} / \mathrm{F}_{\mathrm{o}} / \mathrm{s}$ and KS1-iPSC-derived cardiomyocytes: $0.001 \pm 0.00 \mathrm{~F} / \mathrm{F}_{\mathrm{o}} / \mathrm{s}$, vs. H7-ESC-derived cardiomyocytes: $0.023 \pm 0.002 \mathrm{~F} / \mathrm{F}_{\mathrm{o}} / \mathrm{s}$, and HES3-hESC-derived cardiomyocytes: $0.028 \pm 0.01 \mathrm{~F} / \mathrm{F}_{\mathrm{o}} / \mathrm{s}$; $p<0.05, n=10$; Fig. $4 \mathrm{c}$ ) as well as maximal upstroke (IMR90 iPSC-derived cardiomyocytes: $-0.003 \pm 0.00 \mathrm{~F} / \mathrm{F}_{\mathrm{o}} / \mathrm{s}$, and KS1-iPSC-derived cardiomyocytes: $-0.001 \pm 0.00 \quad \mathrm{~F} / \mathrm{F}_{\mathrm{o}} / \mathrm{s}$, vs. H7-ESC-derived cardiomyocytes: $-0.007 \pm 0.00 \mathrm{~F} / \mathrm{F}_{\mathrm{o}} / \mathrm{s}$ and HES3-hESC-derived cardiomyocytes: $-0.007 \pm 0.00$ $\mathrm{F} / \mathrm{F}_{\mathrm{o}} / \mathrm{s} ; p<0.05, n=10 ;$ Fig. $\left.4 \mathrm{~d}\right)$. The caffeine induced calcium release in suggested the presence of functional SR in hiPSC-derived cardiomyocytes; however, the slower rate of calcium release may imply an impaired calcium release through RyR. Consistently, the application of ryanodine $(10 \mu \mathrm{M})$ resulted in a smaller reduction in amplitude in hiPSC-derived cardiomyocytes (IMR-90 iPSC-derived cardiomyocytes: $16.75 \%$, and KS1-iPSCderived cardiomyocytes: $5.15 \%$, vs. H7-ESC-derived cardiomyocytes: $47.95 \%$, and HES3-hESC-derived cardiomyocytes: $63.18 \%, p<0.05, n=10)$ than human ESCderived cardiomyocytes (Fig. 5b-d). Taken together, these observations suggested the presence of functional internal calcium store with immature calcium release mechanisms.

To further compare the temporal and spatial patterns of calcium transients of hiPSC-derived and hESC-derived cardiomyocytes, transverse line-scan images of calcium transient were performed. As we previously reported in mouse ESC-derived cardiomyocytes [14], both hESC-derived and hiPSC-derived cardiomyocytes exhibited a U-shape calcium wave fronts as in typical feature of t-tubule-deficit cardiomyocytes: the rise of calcium in the periphery was faster than their cell centers (Fig. 6a and b).

Furthermore, in addition to the rhythmic whole-cell calcium transient, there were more high-frequency submaximal-amplitude calcium transients observed in hiPSCderived cardiomyocytes than human ESC-derived cardiomyocytes $(25 \%$ vs. $8.3 \%, p<0.05,(n=25))$. These sub-maximal 


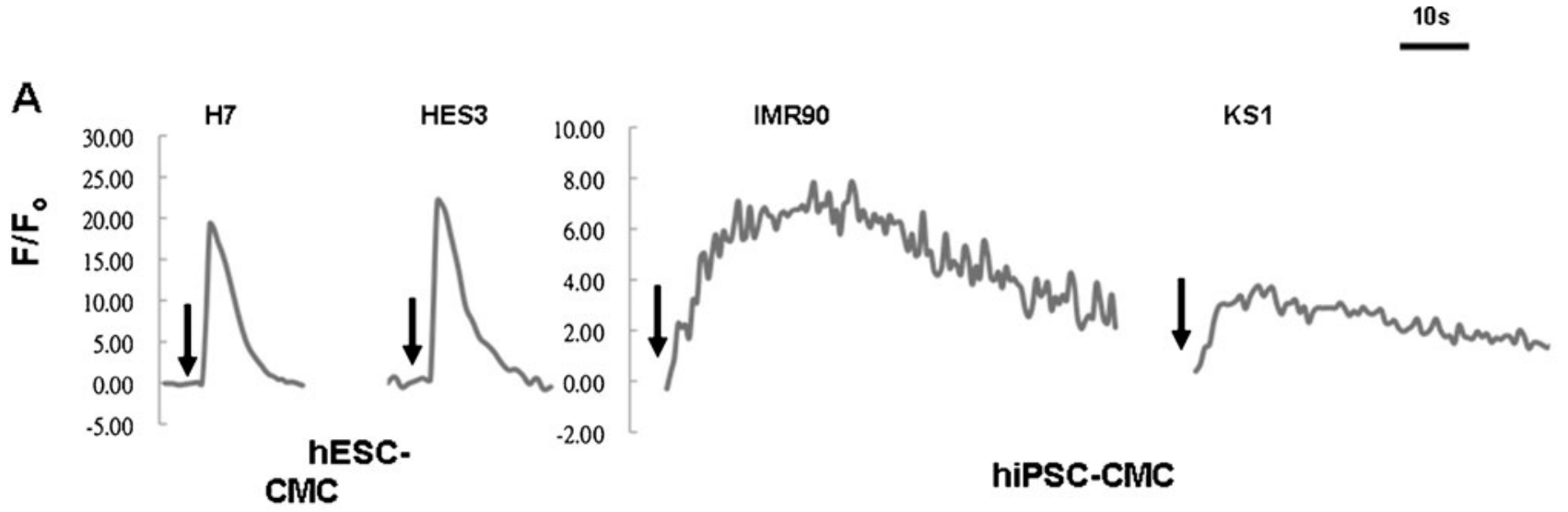

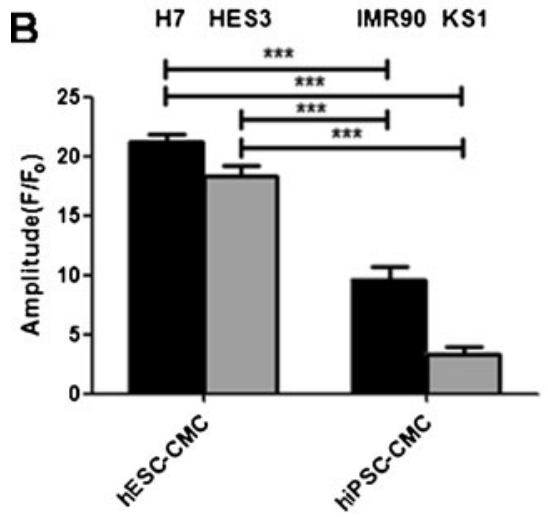
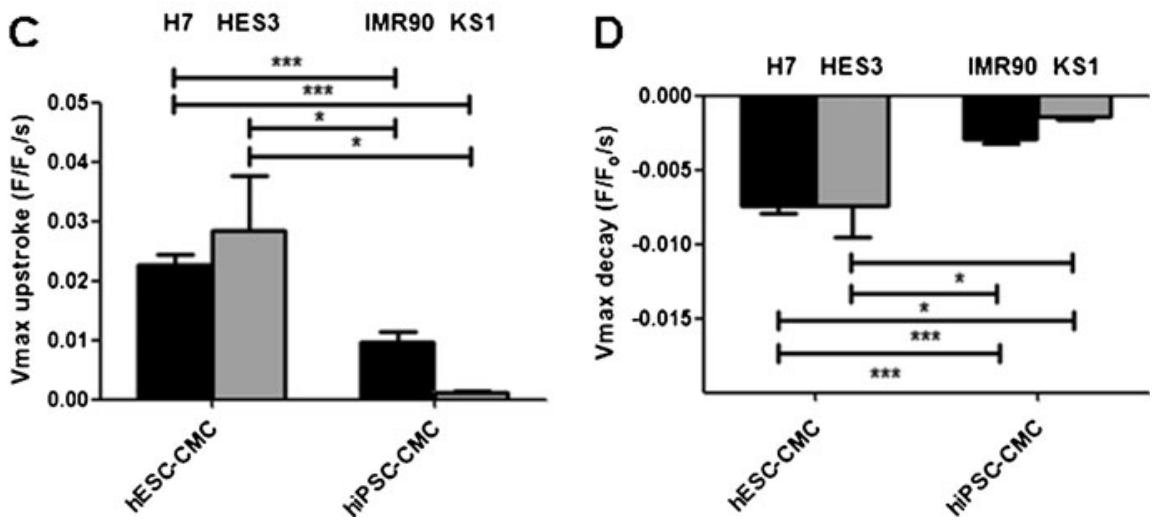

Fig. 4 Representative tracing of caffeine-induced calcium release in a H7- \& HES3-hESC derived cardiomyocytes (H7 \& HES3 hESCCMCs), IMR90- \& KS1-hiPS derived cardiomyocytes (IMR90 \& KS1 hiPSC-CMCs). b Amplitude, c maximal upstroke velocity ( $V_{\max }$ upstroke), and d maximal decay velocity ( $V_{\max }$ decay) of calcium

calcium transients represent local calcium events instead of a whole-cell event. hiPSC-derived cardiomyocytes exhibited more local calcium events than their human ESC counterpart, suggesting a less effective calcium induced calcium release mechanism.

\section{Expression of Calcium Handing Proteins}

To decipher the molecular basis for the difference in calcium handling properties between hiPSC- and hESCderived cardiomyocytes, the expressions of three calcium handling proteins (sodium calcium exchanger-1 (NCX1), RyR2, and SERCA2a) as well as the three cardiac SR luminal auxiliary proteins (calsequestrin, junctin, and triadin), which form a protein complex associated with RyR2 were quantified (Fig. 6d and e). As shown in quantitative reverse transcription polymerase chain reaction, expressions of RyR2 and SERCA2a was significantly lower in two cell lines of hiPSC-derived cardiomyocytes in compared transients in the cardiomyocytes. Unpaired $t$-test was performed between $\mathrm{H} 7$ or HES3 hES-CMCs and the other two lines of iPSCMCs $(n=10 ; \mathrm{n}$ is corresponds to different individual cells derived from three different experiments; ${ }^{*} p<0.05$, and ${ }^{* * *} p<0.0001$ ). Arrow heads indicated the time points that caffeine was added

to $\mathrm{hESC}$ counterpart $(p<0.0005 ; n=3)$ (Fig. $6 \mathrm{~d})$. In addition, among the three cardiac SR luminal auxiliary proteins, the expression of triadin and junctin were significantly lower in cardiomyocytes $(p<0.0005)$, while no significant differences in calsequestrin expressions was observed between the two cell lines (Fig. 6e).

\section{Discussion}

In this study, we report the measurement of whole-cell calcium transients as well as the temporal and spatial properties of cellular calcium gradients in IMR-90 and KS1-hiPSC-derived cardiomyocytes and compared with those of H7- and HES3-hESC-derived cardiomyocytes. The major two findings are as follow. First, although hiPSC-derived cardiomyocytes resembling to hESC counterpart express major SR-calcium handling proteins includhiPSC-derived cardiomyocytes compared with hESC-derived 
A Control

Ryanodine

Control

Ryanodine

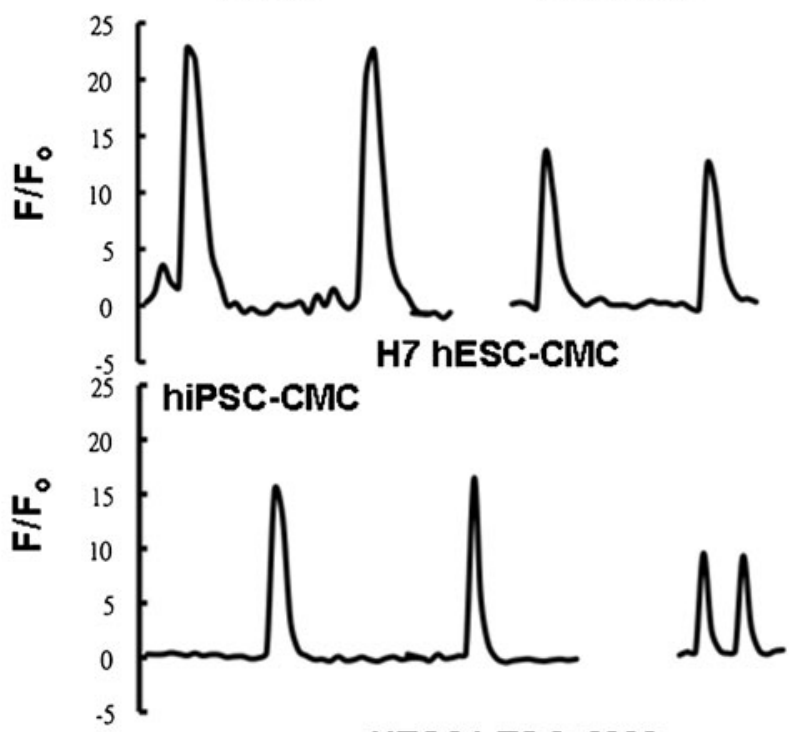

HES3 hESC-CMC
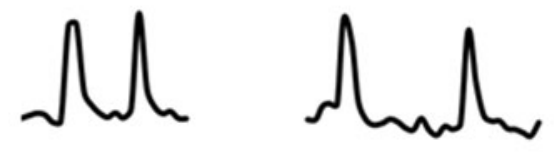

KS1 hiPSC-
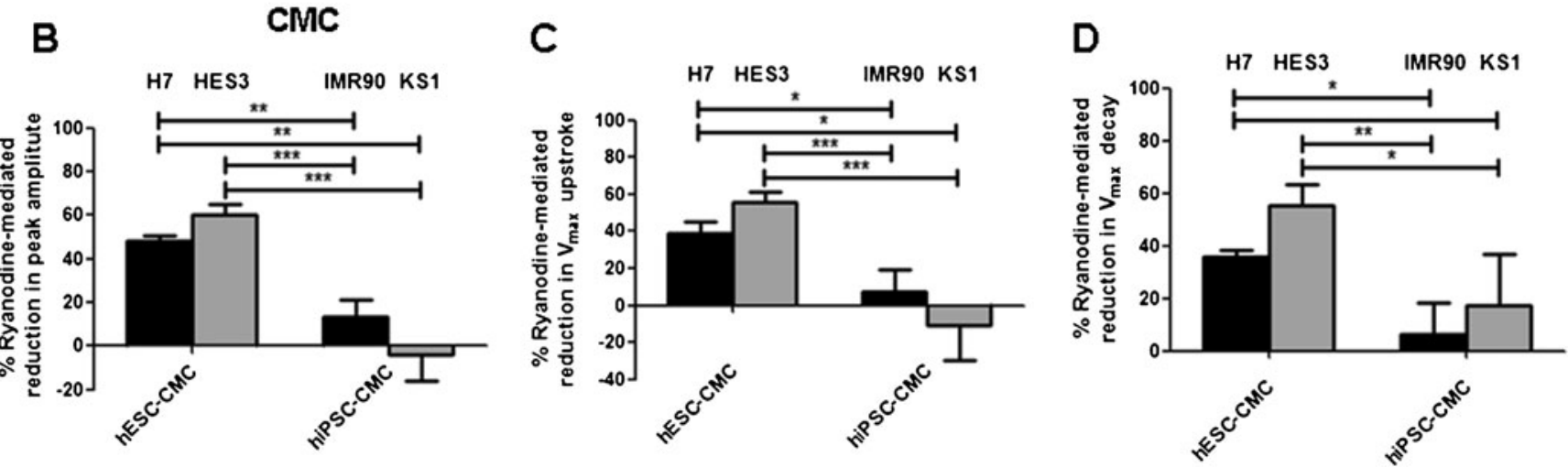

Fig. 5 Representative tracing of ryanodine sensitive calcium release in a H7- \& HES3-hES derived cardiomyocytes (H7- \& HES3-hES CMCs) and IMR90- \& KS1-hiPSC-derived cardiomyocytes (IMR90\& KS1-hiPS CMCs). b Amplitude, $\mathbf{c}$ maximal upstroke velocity ( $V_{\max }$ upstroke), and d maximal decay velocity ( $V_{\max }$ decay) of calcium

ing RyR2, SERCA2a, and NCX-1 and exhibit typical whole-cell calcium transients that respond to caffeine and ryanodine, the CICR mechanism appears not as efficient as that of hESC-derived cardiomyocytes, indicating more immature SR-calcium handling. Second, in addition to more immature whole cell calcium transients, the spatial inhomogeneities in the temporal properties of calcium transients across the width of cardiomyocytes were more pronounced in hiPSC-derived cardiomyocytes than hESC counterpart.

Cardiomyocytes are the cellular workhorse for cardiac contractile function. Their loss in myocardial infarction is irreversible due to their limited regenerative capacity and often results in heart failure. Although cell-based therapy has been envisioned as a therapeutic strategy to regenerate myocardium, transplantation of immature cardiomyocytes transients in the cardiomyocytes. Unpaired $t$-test was performed between two different groups as indicted by arrows in the figure ( $n=12 ; n$ is corresponds to different individual cells derived from three different experiments; ${ }^{*} p<0.05$ and $* * * p<0.0001$ )

results not only poor graft-host integration but may also lead to potential lethal arrhythmia, [27, 28] thereby functional characterization of the stem cell-derived cardiomyocytes represents the crucial first step toward ultimate clinical application. Recently, the electrophysiological properties of hiPSC-derived cardiomyocytes have recently been reported; $[5,6,8,9]$ the calcium handling properties of hiPSC-derived cardiomyocytes have not been studied in detail. In fact, the dynamic alteration of cytosolic calcium concentration plays the central role in cardiac excitationcontraction coupling. Upon membrane depolarization, the influx of calcium via L-type calcium channels triggers calcium release from the internal calcium store, SR via RyRs, which in turn initiates the mechanical contraction [13]. After reaching the peak calcium transient, cytosolic calcium is actively removed from the cytosol, mainly 
Fig. 6 Transverse line-scan confocal of hiPSC- and hESC-derived cardiomyocytes. Representative tracings of temporal and spatial patterns of calcium transients of hiPSCderived cardiomyocytes (a) and $\mathbf{b}$ hESC-derived cardiomyocytes, $\mathbf{c}$ Time delay in the initial calcium rise between cell center and cell periphery in hiPSC- and hESC-derived cardiomyocytes, and $\mathbf{d}$ Quantitation of gene expression of calcium handling proteins of differentiated $\mathrm{H} 7 \mathrm{hES}$ and IMR90-iPS (a) and HES3 hES and KS1-iPS (b). The fold of change was normalized to the cardiac marker, Nkx2.5 expression. Sodium-calcium exchanger-1 (NCX-1), ryanodine receptor 2 (RyR2), sarcoplasmic recticulum calcium-Ca ${ }^{2+}$ ATPase 2a (SERCA2a), calsequestrin (CASQ2), junction (Jun) and triaden (Trd). Unpaired $t$-test was performed between differentiated $\mathrm{H} 7 \mathrm{hESCs}$ vs. IMR90-iPSCs and HES3 hESCs vs. KS1-iPSCs $(n=3 ; * p<0.05$, $* * p<0.005$, and $* * * p<0.0005)$
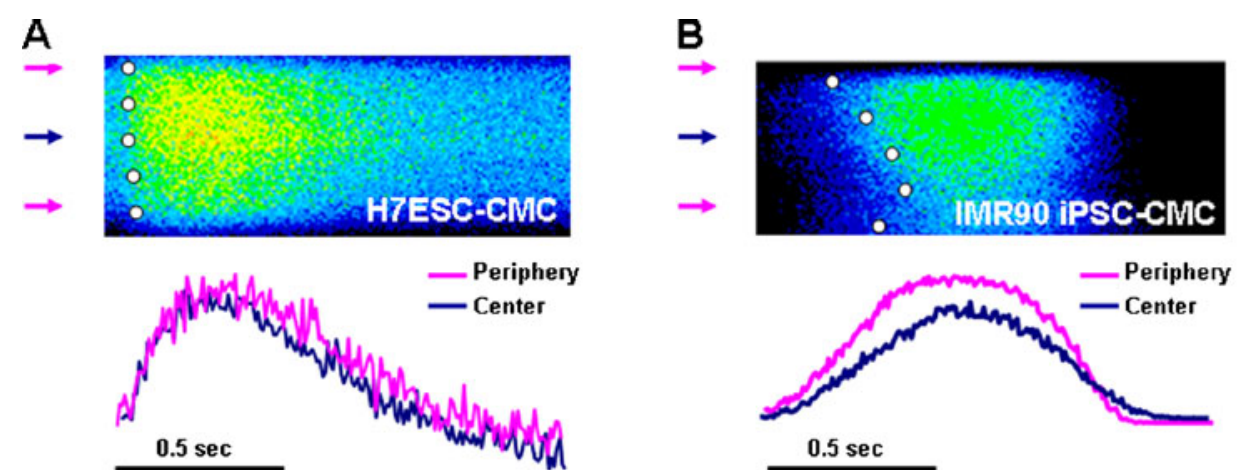

C

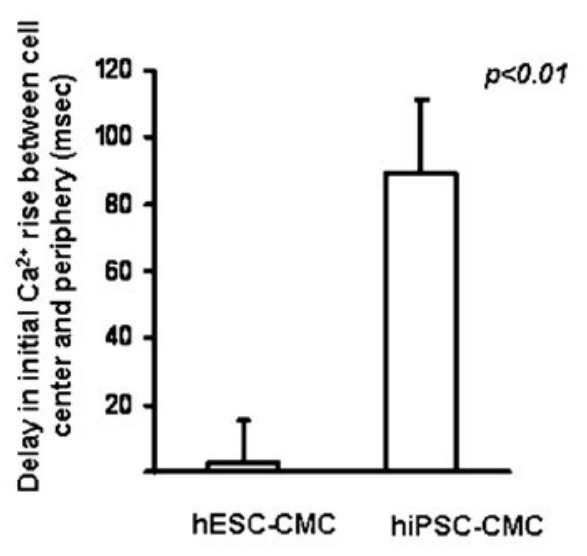

D

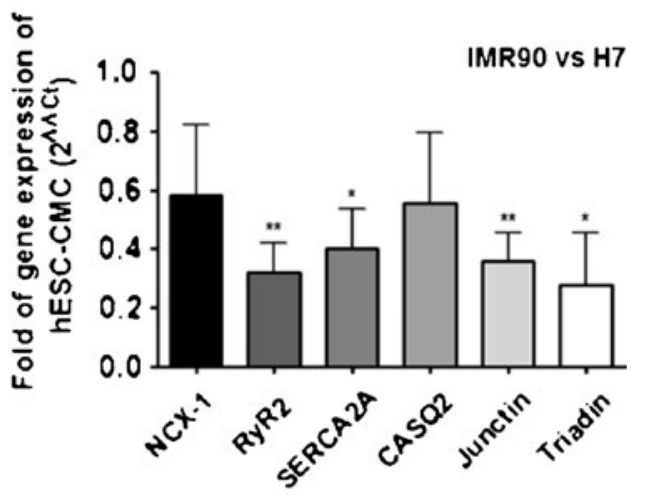

E

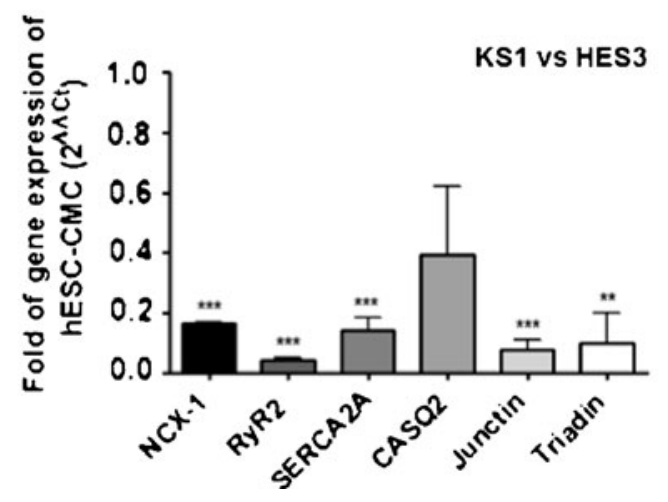

through SERCA-2a back into SR and NCX-1 out of cells [18]. However, it was shown as early as in 2005 by Dolnikov and co-workers that the functional properties related to intracellular calcium handling of hESC-derived cardiomyocytes differ significantly from adult cardiomyocytes due to immature SR capacity [11]. In fact, cardiomyocytes derived from hESC exhibit immature calcium handling properties resembling to that of fetal cardiomyocytes, albeit expressing functional SR [22]. Specifically, hESC-derived cardiomyocytes, though responsive to caffeine and ryanodine, exhibited smaller whole-cell peak amplitude, slower upstroke and decay in calcium transient in comparison to adult cardiomyocytes [10, 11, 22]. In our recordings, while spontaneous rhythmic calcium transients could be readily observed in hiPSC-derived cardiomyocytes, but these calcium transients were of even smaller amplitude, slower upstroke velocity, and slower reuptake compared with H7-hESC-derived cardiomyocytes. In addition, hiPSC-derived cardiomyocytes were less responsive to the application of ryanodine, indicating immature functioning SR relative to hESC counterpart. This may be partly related to the lower expression levels of major calcium handling proteins including SERCA2a and NCX-1 in hiPSC-derived cardiomyocytes. 
Furthermore, in adult cardiomyocytes, RyRs are brought in close proximity to L-type calcium channels at the ttubular system, allowing calcium wavefronts to quickly penetrate to the interior of the cells. In a stark contrast, ESC-derived cardiomyocytes exhibit spatial inhomogeneity in temporal properties of calcium transients across the width of the cells [14, 21]. Specifically, calcium wavefronts initiate earlier and reach the peak faster at the periphery of the ESC-derived cardiomyocytes than the center. As a result, the calcium wavefronts across the width of the cells as revealed by transverse line scan image typically show a U-shaped rise with a marked delay in peak between the cell periphery and the cell center $[14,21]$. Recently, this inhomogeneity in the calcium release wavefront that reduces the efficacy of the calcium induced excitationcontraction coupling, have also been demonstrated in adult cardiomyocytes from failing heart [29-31]. In the present study, while both hiPSC- and hESC-derived cardiomyocytes exhibited this typical U-shaped rise in calcium wavefronts across the width of the cells, the delay between the cell periphery and the cell center was significantly longer in hiPSC-derived cardiomyocytes than hESCcounterpart. It has been suggested that this poor coupling between the calcium influx through L-type calcium channels and the calcium release from the SR through RyRs is secondary to the spatial separation of these ultrastructural organizations due to the lack of t-tubule [14, 21, 29-31]. At the $\mathrm{t}$-tubule, triadin, a $32 \mathrm{kDa}$ protein together with a set of interrelated and interacting proteins including junctin and calsequestrin forms complex with RyRs on the SR and the juxtaposed L-type calcium channels in the sarcolemma. In addition to serve as a physical linker between RyRs and Ltype calcium channels, triadin has also been shown to regulate calcium release via modulating the gating function of RyRs [32], as well as modifying the SR structure [33]. In fact, in triadin null hearts, the ablation of triadin results in significant reduction in junctional SR proteins including RyR2, calsequestrin, and junctin, and 50\% reduction in the contacts between junctional SR and t-tubules, which translate into reduced SR calcium release and impaired negative feedback of SR calcium release [34, 35]. In the present study, the expression of triadin was significantly lower in hiPSC-derived cardiomyocytes than that of hESC counterpart, which may partly explain the immature whole-cell calcium handling properties as well as the temporal inhomogeneity in calcium transient. As transplantation of immature cardiomyocytes results not only poor graft-host integration but also lethal arrhythmia [27, 28 ], in vitro manipulation of stem cell-derived cardiomyocytes is needed in order to yield mature cardiomyocytes. Exploiting various hypertrophic stimuli including triiodonthyronine, ouabain, apoA-I, and hypoxia, we have previously shown that calcium handling properties of
ESC-derived cardiomyocytes could be driven more mature (or adult-like) for potential therapeutic use, and such changes were associated with the upregulation of major calcium handling proteins such in ESC-derived cardiomyocytes [16, 17, 24, 25]. The possibility of driven maturation of hiPSC-derived cardiomyocytes with these strategies requires further investigation.

Our study is limited by certain technological shortcomings. First, the confocal calcium imaging was not performed simultaneously with action potential recordings using patch clamp, thereby the chamber-identity of individual cardiomyocytes cannot be ascertained. Second, the calcium imaging was performed with Fluo-3 instead of ratiometric calcium indicators like Indo-1 or Fura-2, thus precluding the possibility of measuring the absolute intracellular calcium concentration.

Taken collectively, hiPSC-derived cardiomyocytes possess functional but immature SR. This finding has potential clinical implication. Although hiPSC-derived cardiomyocytes, avoiding the potential immune rejection and ethic issues peculiar to $\mathrm{hESC}$, hold promise for cardiac regeneration, the immature calcium-handling properties of hiPSCderived cardiomyocytes can result in poor functional integration and/or lethal arrhythmia after cellular transplantation, thus limiting their potential therapeutic applications. Thereby, it is highly desirable to develop strategies to drive functional maturation ex vivo.

Disclosure summary The authors have nothing to disclose.

Open Access This article is distributed under the terms of the Creative Commons Attribution Noncommercial License which permits any noncommercial use, distribution, and reproduction in any medium, provided the original author(s) and source are credited.

\section{References}

1. Park, I. H., Zhao, R., West, J. A., et al. (2008). Reprogramming of human somatic cells to pluripotency with defined factors. Nature, 451, 141-146.

2. Takahashi, K., Tanabe, K., Ohnuki, M., et al. (2007). Induction of pluripotent stem cells from adult human fibroblasts by defined factors. Cell, 131, 861-872.

3. Yamanaka, S. (2007). Strategies and new developments in the generation of patient-specific pluripotent stem cells. Cell Stem Cell, 1, 39-49.

4. Yu, J., Vodyanik, M. A., Smuga-Otto, K., et al. (2007). Induced pluripotent stem cell lines derived from human somatic cells. Science, 318, 1917-1920.

5. Zhang, J., Wilson, G. F., Soerens, A. G., et al. (2009). Functional cardiomyocytes derived from human induced pluripotent stem cells. Circulation Research, 104, e30-e41.

6. Zwi, L., Caspi, O., Arbel, G., et al. (2009). Cardiomyocyte differentiation of human induced pluripotent stem cells. Circulation, 120, 1513-1523. 
7. Siu, C. W., Liao, S. Y., Liu, Y., et al. (2010). Stem cells for myocardial repair. Thrombosis Haemostasis, 104, 6-12.

8. Gai, H., Leung, E. L., Costantino, P. D., et al. (2009). Generation and characterization of functional cardiomyocytes using induced pluripotent stem cells derived from human fibroblasts. Cell Biology International, 33, 1184-1193.

9. Yokoo, N., Baba, S., Kaichi, S., et al. (2009). The effects of cardioactive drugs on cardiomyocytes derived from human induced pluripotent stem cells. Biochemical and Biophysical Research Communications, 387, 482-488.

10. Dolnikov, K., Shilkrut, M., Zeevi-Levin, N., et al. (2005). Functional properties of human embryonic stem cell-derived cardiomyocytes. Annals of the New York Academy of Sciences, 1047, 66-75.

11. Dolnikov, K., Shilkrut, M., Zeevi-Levin, N., et al. (2006). Functional properties of human embryonic stem cell-derived cardiomyocytes: intracellular $\mathrm{Ca} 2+$ handling and the role of sarcoplasmic reticulum in the contraction. Stem Cells, 24, 236-245.

12. Fu, J. D., Li, J., Tweedie, D., et al. (2006). Crucial role of the sarcoplasmic reticulum in the developmental regulation of $\mathrm{Ca} 2+$ transients and contraction in cardiomyocytes derived from embryonic stem cells. The FASEB Journal, 20, 181-183.

13. Bers, D. M. (2008). Calcium cycling and signaling in cardiac myocytes. Annual Review of Physiology, 70, 23-49.

14. Au, K. W., Liao, S. Y., Lee, Y. K., et al. (2009). Effects of iron oxide nanoparticles on cardiac differentiation of embryonic stem cells. Biochemical and Biophysical Research Communications, 379, 898-903.

15. Cluzeaut, F., \& Maurer-Schultze, B. (1986). Proliferation of cardiomyocytes and interstitial cells in the cardiac muscle of the mouse during pre- and postnatal development. Cell and Tissue Kinetics, 19, 267-274.

16. Lee, Y. K., Ng, K. M., Chan, Y. C., et al. (2010). Triiodothyronine promotes cardiac differentiation and maturation of embryonic stem cells via the classical genomic pathway. Molecular Endocrinology, 24, 1728-1736.

17. Lee, Y. K., Ng, K. M., Lai, W. H., et al. (2011). Ouabain facilitates cardiac differentiation of mouse embryonic stem cells through ERK1/2 pathway. Acta Pharmacologica Sinica, 32, 52-61.

18. Satin, J., Itzhaki, I., Rapoport, S., et al. (2008). Calcium handling in human embryonic stem cell-derived cardiomyocytes. Stem Cells (Dayton, Ohio), 26, 1961-1972.

19. Lai, W. H., Ho, J. C., Lee, Y. K., et al. (2010). ROCK inhibition facilitates the generation of human-induced pluripotent stem cells in a defined, feeder-, and serum-free system. Cellular Reprogramming, 12, 641-653.

20. Yang, L., Soonpaa, M. H., Adler, E. D., et al. (2008). Human cardiovascular progenitor cells develop from a KDR+ embryonicstem-cell-derived population. Nature, 453, 524-528.

21. Lieu, D. K., Liu, J., Siu, C. W., et al. (2009). Absence of transverse tubules contributes to non-uniform $\mathrm{Ca}(2+)$ wavefronts in mouse and human embryonic stem cell-derived cardiomyocytes. Stem Cells and Development, 18, 1493-1500.
22. Liu, J., Fu, J. D., Siu, C. W., et al. (2007). Functional sarcoplasmic reticulum for calcium handling of human embryonic stem cellderived cardiomyocytes: insights for driven maturation. Stem Cells, 25, 3038-3044.

23. Liu, J., Lieu, D. K., Siu, C. W., et al. (2009). Facilitated maturation of $\mathrm{Ca} 2+$ handling properties of human embryonic stem cell-derived cardiomyocytes by calsequestrin expression. American Journal of Physiology. Cell Physiology, 297, C152-C159.

24. Ng, K. M., Lee, Y. K., Lai, W. H., et al. (2011). Exogenous expression of human apoA-I enhances cardiac differentiation of pluripotent stem cells. PLoS One, 6, e19787.

25. Ng, K. M., Lee, Y. K., Chan, Y. C., et al. (2010). Exogenous expression of HIF-1 alpha promotes cardiac differentiation of embryonic stem cells. Journal of Molecular and Cellular Cardiology, 48, 1129-1137.

26. Ho, J. C., Zhou, T., Lai, W. H., et al. (2011). Generation of induced pluripotent stem cell lines from 3 distinct laminopathies bearing heterogeneous mutations in lamin A/C. Aging (Albany NY), 3, 380-390.

27. Liao, S. Y., Liu, Y., Siu, C. W., et al. (2010). Proarrhythmic risk of embryonic stem cell-derived cardiomyocyte transplantation in infarcted myocardium. Heart Rhythm, 7, 1852-1859.

28. Zhang, Y. M., Hartzell, C., Narlow, M., et al. (2002). Stem cellderived cardiomyocytes demonstrate arrhythmic potential. Circulation, 106, 1294-1299.

29. He, J., Conklin, M. W., Foell, J. D., et al. (2001). Reduction in density of transverse tubules and L-type $\mathrm{Ca}(2+)$ channels in canine tachycardia-induced heart failure. Cardiovascular Research, 49, 298-307.

30. Louch, W. E., Bito, V., Heinzel, F. R., et al. (2004). Reduced synchrony of $\mathrm{Ca} 2+$ release with loss of T-tubules-a comparison to $\mathrm{Ca} 2+$ release in human failing cardiomyocytes. Cardiovascular Research, 62, 63-73.

31. Song, L. S., Sobie, E. A., McCulle, S., et al. (2006). Orphaned ryanodine receptors in the failing heart. Proceedings of the National Academy of Sciences of the United States of America, 103, 4305-4310

32. Terentyev, D., Cala, S. E., Houle, T. D., et al. (2005). Triadin overexpression stimulates excitation-contraction coupling and increases predisposition to cellular arrhythmia in cardiac myocytes. Circulation Research, 96, 651-658.

33. Marty, I., Faure, J., Fourest-Lieuvin, A., et al. (2009). Triadin: what possible function 20 years later? Journal de Physiologie, 587, 3117-3121.

34. Knollmann, B. C. (2009). New roles of calsequestrin and triadin in cardiac muscle. Journal de Physiologie, 587, 3081-3087.

35. Chopra, N., Yang, T., Asghari, P., et al. (2009). Ablation of triadin causes loss of cardiac $\mathrm{Ca} 2+$ release units, impaired excitationcontraction coupling, and cardiac arrhythmias. Proceedings of the National Academy of Sciences of the United States of America, 106, 7636-7641. 